\title{
Quantum Networks with Chiral-Light-Matter Interaction in Waveguides
}

\author{
Sahand Mahmoodian, Peter Lodahl, and Anders S. Sørensen \\ Niels Bohr Institute, University of Copenhagen, Blegdamsvej 17, DK-2100 Copenhagen, Denmark
}

(Received 29 February 2016; published 5 December 2016)

\begin{abstract}
We propose a scalable architecture for a quantum network based on a simple on-chip photonic circuit that performs loss-tolerant two-qubit measurements. The circuit consists of two quantum emitters positioned in the arms of an on-chip Mach-Zehnder interferometer composed of waveguides with chiral-light-matter interfaces. The efficient chiral-light-matter interaction allows the emitters to perform high-fidelity intranode two-qubit parity measurements within a single chip and to emit photons to generate internode entanglement, without any need for reconfiguration. We show that, by connecting multiple circuits of this kind into a quantum network, it is possible to perform universal quantum computation with heralded two-qubit gate fidelities $\mathcal{F} \sim 0.998$ achievable in state-of-the-art quantum dot systems.
\end{abstract}

DOI: 10.1103/PhysRevLett.117.240501

The overarching goal of modern quantum physics is to construct large-scale quantum networks [1] where the evolution and interaction of its constituents can be controlled at the quantum level. Such systems would provide a platform to simulate arbitrary quantum systems [2], perform quantum computation [3], and provide provably secure information transfer [4]. Building a monolithic quantum system with a large number of qubits is a very challenging task, while controlled quantum operations on a small number of qubits with high fidelity is a feasible objective. An attractive approach to scale to a large quantum system is thus to construct a large number of few-qubit nodes and connect them with optical links [5-8]. In such systems, it has been shown that quantum processing can successfully proceed even if the optical links are noisy $[6,7]$. Building a scalable network of such few-qubit nodes becomes possible if one can combine an efficient lightmatter interface with high-fidelity quantum operations between qubits within a single node.

In recent years, quantum nanophotonic systems have matured greatly, and photonic nanostructures have been interfaced efficiently with quantum dots $[9,10]$, atoms $[11,12]$, and diamond color centers [13]. In particular, photons in photonic-crystal waveguides (PCWs) can be interfaced with quantum dots (QDs) with near-unity efficiency [14] and can also exhibit chiral-light-matter interaction where optical transitions with opposite helicity couple efficiently to opposite directions [15-18]. A multitude of two-qubit gates have also been proposed for photon-emitter systems; however, there is no clear path for how to scale these ingredients to build large networks. In particular, since most schemes involve photon transfer between qubits to implement gates over long distances, it is unclear how to scale the systems without an on-chip reconfiguration and/or lossy in- or outcoupling from chips.

In this work, we propose an architecture for realizing a quantum network based on simple elements. To this end, we develop a novel scheme to measure the parity of two quantum dots' spin state in a manner that is robust to loss and achieve near-unity fidelity even for imperfect couplings. By linking many copies of these circuits together, we are able to realize a quantum network capable of universal quantum computation. The layout is shown in Figs. 1(a) and 1(b) and consists of two QDs embedded in the arms of an on-chip Mach-Zehnder interferometer (MZI), with an additional single-photon source. Here, dielectric waveguides couple to form beam splitters and are integrated with on-chip photon detectors. The waveguides are coupled to chiral elements $\chi$ [Fig. 1(a)], each of which contains a quantum emitter in a waveguide with a chiral-light-matter interaction. The modes of this waveguide have electric fields with in-plane circular polarization where counterpropagating modes have countercirculating polarizations $[12,15]$. In our proposal, the chiral element is a QD coupled to an engineered PCW [15], but other systems such as atoms coupled to whispering gallery mode resonators [12] can also be used. The logical qubits are formed by the spin ground states of the QDs, $|\downarrow\rangle \equiv|0\rangle$ and $|\uparrow\rangle \equiv|1\rangle$, and the optical transitions are used for both gate operations and communication. A chiral-light-matter interaction gives two important advantages. (i) It provides an efficient way to separate two optical transitions with opposite helicity, allowing each to be used for a distinct purpose. Here we use the right-hand circularly polarized $\left(\sigma_{+}\right)$transition for local operations and the left-hand circularly polarized $\left(\sigma_{-}\right)$transition for internode communication [Fig. 1(c)]. (ii) Our scheme requires a photon to coherently scatter off a quantum emitter and acquire a conditional phase shift before being passed on to the next element. The use of a chiral interaction allows this to be achieved with near-unity fidelity in a simple passive structure without active switching. Similar functionality could, in principle, also be achieved with circulators or by exploiting the polarization in single-sided structures 
(a) Chiral element

\section{(b) Network node}

(i) Intranode parity measurement

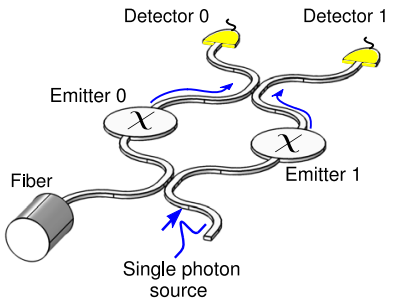

(ii) Internode entanglement

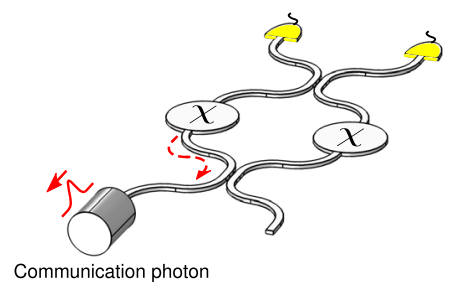

(c) Linked Nodes

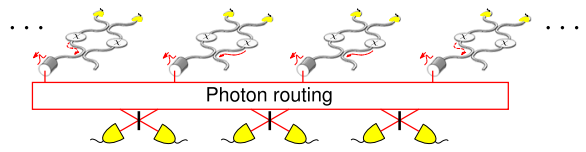

FIG. 1. Architecture for a quantum network. (a) The chiral element $\chi$ is composed of a quantum dot coupled to a photonic crystal waveguide. The quantum dot's optical transitions are circularly polarized with spin ground states that act as logical qubits, and the waveguide is engineered to have a chiral-lightmatter interaction [15]. A $\sigma_{+}\left(\sigma_{-}\right)$polarized optical transition couples to the right (left) propagating waveguide mode. The electric field intensity emitted from a $\sigma_{+}$dipole is shown with bright (dark) colors representing high (low) intensity. (b) The building block of the quantum network consists of two chiral elements $\chi$ in the arms of an interferometer with photon detectors at the output. (i) A two-qubit parity measurement within a node is performed using a single photon resonant on the $\sigma_{+}$transition scattering off the emitters. The parity of the emitters' spin states determines the phase difference of the interferometer and thus the output path of the photon. (ii) Photon emission from the $\sigma_{-}$transition is used to link nodes through an optical fiber. (c) Photons are routed to mix with the output from another node with photon detection heralding entanglement between any two nodes.

supporting circular polarization. Such elements would, however, be difficult to implement on chip.

Information is processed within a node using a singlephoton source which sends a photon resonant with the $\sigma_{+}$ polarized transition into the MZI from the bottom-right arm [see Fig. 1(b)(i)]. This ancilla photon interacts with the two emitters and, as we detail below, measures the parity $[19,20]$ of the two qubits upon detection; i.e., it returns 0 for $|00\rangle$ and $|11\rangle$ and 1 for $|01\rangle$ and $|10\rangle$ and projects the system into one of these subspaces. Since the operation is heralded by a photon detection, we show that losses act to lower the success probability of the measurement rather than its fidelity. Finally, we show that such a parity measurement integrated in a simple passive circuit enables entanglement generation, swapping, and distillation, as well as teleportation-based gates. These operations constitute a complete toolbox to perform universal quantum computation across a quantum network.

We now consider the operation of the circuit in Fig. 1(b)(i) for performing a parity measurement. The chiral coupling of the emitter to the waveguide is quantified by the directional $\beta$ factor of the $\sigma_{+}$polarized optical transition to the rightward propagating waveguide mode $\beta=\Gamma_{R} /\left(\Gamma_{R}+\Gamma_{L}+\gamma\right)$, where $\Gamma_{R}$ and $\Gamma_{L}$ are the respective decay rates to the right- and left-propagating waveguide modes and $\gamma$ is the decay rate to radiation modes. In the presence of a detuning $\Delta_{j}$, the transmission coefficient is $t_{j}=1-2 \beta_{j} /\left(1-2 i \beta_{j} \Delta_{j} / \Gamma_{R}^{j}\right)[15,21]$, where the script $j$ denotes emitter 0 [left emitter in Fig. 1(b)(i)] or 1 (right emitter). Ideally, narrow band on-resonance photons $\left(\beta_{j}=1, \Delta_{j}=0\right)$ have unit transmittance and are imparted with a $\pi$ phase shift $\left(t_{j}=-1\right)$ in each arm of the MZI if the emitter is in state $|1\rangle$. The ideal operation of the MZI for measuring parity is then clear: For odd-parity spin states $(|01\rangle$ and $|10\rangle)$ the MZI is imbalanced and photons register clicks on detector 1 , while for even parity $(|00\rangle$ and $|11\rangle)$ the MZI is balanced and clicks are registered on detector 0 . For an arbitrary input pure state $|\Psi\rangle=\sum_{i, j=0,1} c_{i j}|i j\rangle|1\rangle_{\mathrm{ph}}$, where $i$ and $j$ denote the states of the left and right stationary qubits, respectively, the output state is

$$
\begin{aligned}
\mid \text { out }\rangle^{(1)}= & |0\rangle_{\mathrm{ph}}\left[2 c_{00}|00\rangle+\left(t_{0}+t_{1}\right) c_{11}|11\rangle\right] / 2 \\
& +|0\rangle_{\mathrm{ph}}\left[\left(1+t_{1}\right) c_{01}|01\rangle+\left(1+t_{0}\right) c_{10}|10\rangle\right] / 2 \\
& +|1\rangle_{\mathrm{ph}}\left[\left(1-t_{0}\right) c_{10}|10\rangle-\left(1-t_{1}\right) c_{01}|01\rangle\right] / 2 \\
& \left.+|1\rangle_{\mathrm{ph}}\left[\left(t_{0}-t_{1}\right) c_{11}|11\rangle\right] / 2+\mid \text { lost }\right\rangle,
\end{aligned}
$$

where $|0\rangle_{\mathrm{ph}}\left(|1\rangle_{\mathrm{ph}}\right)$ represent a photon in the left (right) arm. Here, $\mid$ lost $\rangle$ is not normalized and describes states where the photon is scattered into modes other than the rightpropagating waveguide mode and is lost. The exact form of this term is not important, as gate successes are heralded by detector clicks. The terms on the first and third lines of (1) are the desired output states for a parity measurement, while the terms on the second and fourth lines of (1) are erroneous results due to imperfections; i.e., the state of the ancilla photon is not equal to the parity of the qubits. The error terms on the second line occur due to imbalance in the MZI caused by loss $\left(\beta_{0}, \beta_{1}<1\right)$ or detuning $\left(\Delta_{0}, \Delta_{1} \neq 0\right)$, while the error term on the fourth line occurs due to inequivalent emitters $\left(t_{0} \neq t_{1}\right)$. For all errors, the probability of getting an erroneous click scales quadratically with its respective error parameter resulting in a very low probability, which scales as, e.g., $(1-\beta)^{2}$, and thus vanishes rapidly as $\beta \rightarrow 1$. The corresponding success probability and infidelity are shown in Figs. 2(a) and 2(b).

If even higher fidelity gate operation is required, errors caused by imbalances in the MZI can be minimized using a two-click protocol: The first photon detection is followed by a $\sigma_{x}$ operation (spin flip) on both qubits, and a second photon is launched into the MZI. Two detection events on 

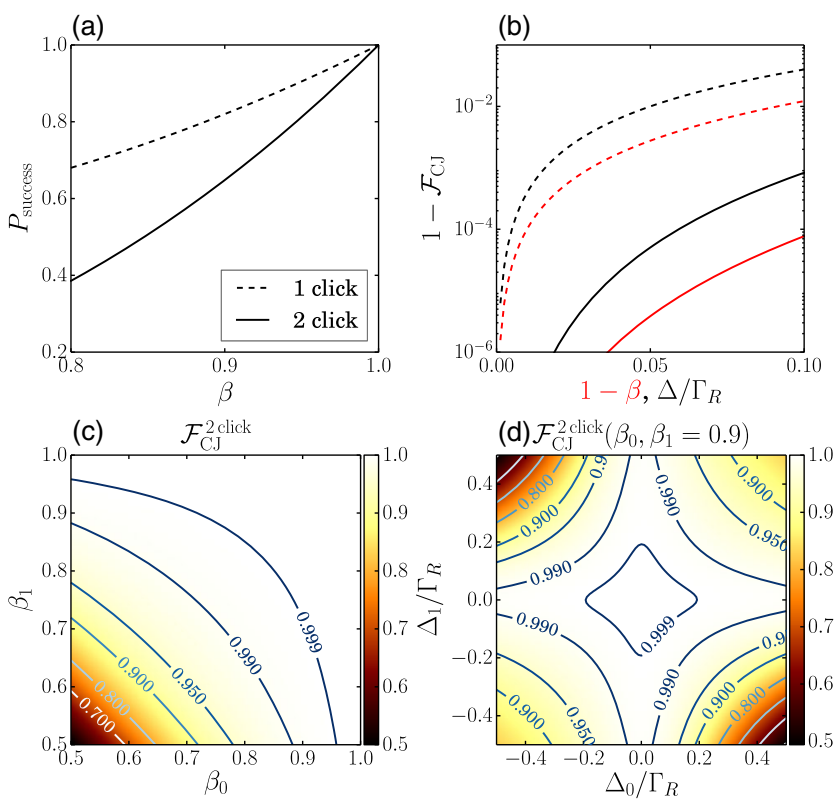

FIG. 2. Analysis of the parity measurement. (a) The success probability versus $\beta$ factor of the emitters $\left(\beta_{0}=\beta_{1}\right)$ for the two protocols. (b) Choi-Jamiołkowski infidelity $1-\mathcal{F}_{\mathrm{CJ}}$ versus $1-\beta$ for resonant emitters with $\beta=\beta_{0}=\beta_{1}$ (light lines) and versus mutual detuning $\Delta=-\Delta_{0}=\Delta_{1}$ (dark lines), for one-click (broken lines) and two-click (solid lines) protocols. Fidelity for two-click protocol $\mathcal{F}_{\mathrm{CJ}}^{\text {2click }}$ versus (c) $\beta_{0}$ and $\beta_{1}$ for resonant emitters and (d) $\Delta_{0} / \Gamma_{R}$ and $\Delta_{1} / \Gamma_{R}$ with $\beta_{0}=\beta_{1}=0.9$.

the same detector heralds success; otherwise, the gate fails. The rotation and second photon scattering has three effects. (i) It reduces the probability of loss and detuning-induced errors to $\sim\left|1+t_{0}\right|^{2}\left|1+t_{1}\right|^{2}$. (ii) It removes the distortion in the states spanned by $|00\rangle$ and $|11\rangle$ caused by different multiplicative factors of $|00\rangle$ and $|11\rangle$ in the first line of (1). (iii) It removes the error term due to inequivalent emitters. The reduced errors come at the expense of a reduced success probability with the two-click protocol requiring two successful detection events. A comparison of the success probabilities for one- and two-click protocols is plotted versus the $\beta$ factor in Fig. 2(a). For detailed calculations, see Supplemental Material [22]. We have also computed the fidelity of our implementation of the parity measurement. We compute the Choi-Jamiolkowski (CJ) fidelity, which can be thought of as an average fidelity of all input states [23,24] (see [22] for more information). Figure 2(b) compares the CJ infidelity for one- and twoclick protocols versus the $\beta$ factor and mutual detuning. The two-click protocol (solid lines) has significantly lower infidelity compared to the one-click protocol (dashed lines). Additionally, Figs. 2(c) and 2(d) show the two-click protocol fidelity for emitters with different $\beta$ factors and detunings. In PCWs, coupling to nonguided radiation modes is suppressed, and QDs can typically have $\beta \gtrsim$ 0.9 [14], which for $\Delta=0$ gives an infidelity $1-\mathcal{F}_{\text {CJ }}^{\text {2click }}<$ $10^{-3}$ for the two-click protocol. The fidelity is, however, more sensitive to detuning. Since the resonance frequencies of the QDs can be tuned electrically, we can expect $\Delta_{1}$, $\Delta_{2}<\Gamma_{R} / 10$, which, for a $\beta$ factor $\beta=0.9$, gives $\mathcal{F}_{\text {CJ }}^{\text {click }} \gtrsim 0.999$.

Until now, we have considered only errors due to loss and detuning. Another source of error is incoherent scattering off the quantum emitter, which for QDs can be induced by pure dephasing and phonon-assisted scattering. These errors scale linearly with the fraction of incoherent scattering (see [22]). Encouragingly, observations of photon emission with up to $99 \%$ indistinguishability [25-27] and lifetime-limited linewidths [28] indicate that the effects of pure dephasing can be limited in QDs. However, phonon-assisted relaxation accounts for $\sim 10 \%$ of the emission in bulk QD samples [26,29]. Operating the gate with high fidelity thus requires filtering out incoherently scattered photons. The spectral sideband associated with phonon-assisted scattering is spread over a width of $\sim 2 \mathrm{~nm}[26,29]$. The zero-phonon line can therefore be selectively filtered using a cavity with quality factor $Q \sim 10^{4}$, which can be integrated on chip [30] and positioned before the single-photon detectors. Once the incoherent light is filtered out, it acts only to lower the success probability and fidelity via a lower $\beta$ factor. Since these errors scale with the fourth power for the two-click protocol, the effect becomes small for phonon-assisted relaxation fractions $\lesssim 10 \%$.

We now consider how the nodes in Fig. 1(b) can be connected to perform a quantum computation. The aim is to perform a teleportation-based quantum computation based on nodes linked via entanglement [6]. To this end, spontaneously emitted photons from the $\sigma_{-}$polarized transition are used for internode communication, since its emission couples to the left-propagating mode. This is shown schematically in Figs. 1(b) and 1(c), where single photons emitted from adjacent nodes couple to output fibers and meet at a beam splitter. Photon detection then heralds the generation of entanglement between the spin states in the two nodes using a time-bin version of the protocol of Refs. [31,32] (see [22]). Since the generation is heralded, it can be attempted until successful. We note that beam splitter 1 has a 50:50 splitting ratio so that half the photons are lost, but its efficiency can be improved by a reconfiguration [33]. Although entanglement can be generated through protocols that are robust to noise, imperfect local operations and gates add infidelity to the Bell states. To improve the quality of entanglement, it is possible to apply entanglement distillation if two nodes $A$ and $B$ share two Bell states [34,35]. A standard entanglement purification protocol [35] can be directly implemented through the parity measurement described above, enabling the generation of high-fidelity Bell states (see [22]).

Once entanglement has been generated between nodes, it is a resource enabling the construction of larger networks. As a particular example, entanglement swapping by 
measuring the qubits within a node in the Bell basis can be used in a quantum repeater architecture to achieve longdistance quantum communications [36]. In the proposed circuits, this can be achieved by measuring parity followed by a rotation and a projective measurement of the spins (see [22]). Here, the projective spin measurement can be performed by scattering photons off the $\sigma_{+}$polarized optical transition from an external laser and using the on-chip detectors to measure the fluorescence.

Alternatively, we can use the generated entanglement between nodes to perform a teleportation-based controlled phase (Cz) gate between distant logical qubits $|a\rangle$ and $|b\rangle$, as shown in Fig. 3(a). In this scheme, one emitter in the MZI plays the role of a resource qubit, forming half of a Bell pair distributed across two nodes, while the other is the logical qubit. The circuit diagram of the CZ gate is shown in Fig. 3(b) and consists of two parts: (i) accumulation of a controlled phase and (ii) a quantum eraser. As shown in Ref. [22], this procedure implements a gate on the logical qubits after successful parity and resource-qubit measurements. The first part involves a parity measurement on each pair at $A$ and $B$ with conditional qubit rotations depending on the measurement outcomes. The quantum eraser then consists of Hadamard rotations $H$ and projective measurements on the resource qubits 1 and 3 with conditional rotations on the logical qubits. Since the parity measurements each operate on different qubits, the fidelity of the $\mathrm{CZ}$ gate scales with the product of the two parity measurement fidelities, i.e., $\mathcal{F}_{\text {CJ }}^{2}$ (see [22]). The construction of a gate between distant qubits along these lines is known to be a sufficient resource for universal quantum computation despite it being probabilistic, since it enables the construction of cluster states [37,38]. At the same time, it is conceivable that more efficient architectures may be

(a)

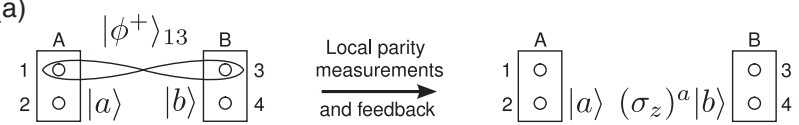

(b)

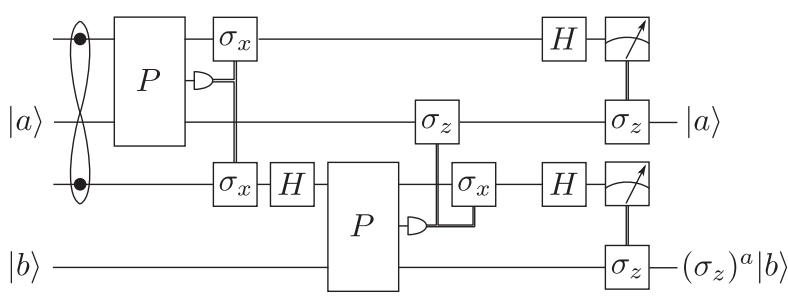

FIG. 3. Teleportation-based CZ gate using parity measurements. (a) Schematic showing that a Bell pair $\left|\phi^{+}\right\rangle_{13}=(|00\rangle+$ $|11\rangle) / \sqrt{2}$ shared between nodes can be used as a resource to perform a teleportation-based $\mathrm{CZ}$ gate between distant qubits $|a\rangle$ and $|b\rangle$. (b) Circuit diagram for the teleportation-based $\mathrm{CZ}$ gate using parity measurements $P$ along with unitary single-qubit rotations represented by the $\sigma_{x}$ and $\sigma_{z}$ Pauli operators conditioned on the measurement outcomes along with Hadamard transformations $H$. constructed by combining it with error-correcting codes designed to correct for qubit loss [39].

We finally consider implementations based on different quantum emitters and photonic platforms. Quantum dots in PCWs have been used to demonstrate near-unity $\beta$ factors [14] with Purcell-enhanced optical transition lifetimes of $\sim 0.2$ ns $[9,14]$. Our implementation is based on coherently scattering photons; therefore, to maintain near-unity fidelity, the single photons need to have temporal widths that are much longer than the emission lifetime, i.e., $\Gamma / \sigma \gtrsim 50$ (see [22]). This means that the optical transition must be free of noise on this time scale. Indistinguishable photon generation through coherent scattering [26] indicates that this is possible with QDs. The photon pulse width sets the minimum time required to measure parity. Since the twoclick protocol requires two scattered photons, the minimum measurement time is $\gtrsim 20 \mathrm{~ns}$, setting a minimum bound on the required coherence time of the spin states of the qubits. Recent work on hole spins in QDs $[40,41]$ is encouraging, but further work is required to examine these properties when the external magnetic field points along the growth direction, which is required to suppress diagonal transitions and make the transition dipoles circularly polarized [42]. Additionally, an integrated photon source that produces photons of the required linewidth is needed. This may be achieved using a QD whose spontaneous emission lifetime is suppressed [43] in comparison to the QDs in the MZI, which can be Purcell enhanced. Alternatively, photons may be produced using Raman fluorescence [44]. Finally, our protocol requires coherent spin manipulation which can also be achieved optically or by using microwave pulses matching the detuning of the spin states. Optical spin manipulation can be implemented in QD molecules, which possess both spin-pumping and cycling transitions [45]. Alternatively, microwave spin resonances have been demonstrated with QDs [46], but coherent manipulation has yet to be achieved.

In conclusion, we have designed and analyzed a simple architecture for a quantum network based on quantum dots. The architecture uses chiral interaction to construct a simple on-chip universal building block which can be merged and scaled to perform universal quantum computation. Our implementation uses stationary qubits for computation and photonic flying qubits for communication and as ancillas to herald gate success. Although we mainly considered QD-based applications, other emitters also have the potential to be used to construct our proposed circuit. Diamond color centers provide an alternative solid-state platform for realizing chiral photonic systems and can be integrated into photonic nanostructures [47]. In particular, silicon vacancy centers' bright zero-phonon line $[48,49]$ makes them a promising candidate. Efficient atom-based chiral-light-matter interaction has also recently been demonstrated [12], making single atoms trapped near photonic nanostructures another potential platform for realizing our proposed gate. 
S. M. acknowledges discussions with Immo Söllner, Petru Tighineanu, Leonardo Midolo, and Alisa Javadi. We gratefully acknowledge financial support from the Villum Foundation, the Carlsberg Foundation, the Danish Council for Independent Research (Natural Sciences and Technology and Production Sciences), and the European Research Council (ERC Consolidator Grants ALLQUANTUM and QIOS).

[1] H. J. Kimble, Nature (London) 453, 1023 (2008).

[2] R. P. Feynman, Int. J. Theor. Phys. 21, 467 (1982).

[3] T. D. Ladd, F. Jelezko, R. Laflamme, Y. Nakamura, C. Monroe, and J. L. O'Brien, Nature (London) 464, 45 (2010).

[4] N. Gisin, G. Ribordy, W. Tittel, and H. Zbinden, Rev. Mod. Phys. 74, 145 (2002).

[5] A. Sørensen and K. Mølmer, Phys. Rev. A 58, 2745 (1998).

[6] J. I. Cirac, A. K. Ekert, S. F. Huelga, and C. Macchiavello, Phys. Rev. A 59, 4249 (1999).

[7] L. Jiang, J. M. Taylor, A. S. Sørensen, and M. D. Lukin, Phys. Rev. A 76, 062323 (2007).

[8] C. Monroe, R. Raussendorf, A. Ruthven, K. R. Brown, P. Maunz, L.-M. Duan, and J. Kim, Phys. Rev. A 89, 022317 (2014).

[9] P. Lodahl, S. Mahmoodian, and S. Stobbe, Rev. Mod. Phys. 87, 347 (2015).

[10] G. Reithmaier, M. Kaniber, F. Flassig, S. Lichtmannecker, K. Müller, A. Andrejew, J. Vučković, R. Gross, and J. J. Finley, Nano Lett. 15, 5208 (2015).

[11] A. Goban, C.-L. Hung, S.-P. Yu, J. D. Hood, J. A. Muniz, J. H. Lee, M. J. Martin, A. C. McClung, K. S. Choi, D. E. Chang, O. Painter, and H. J. Kimble, Nat. Commun. 5, 3808 (2014).

[12] C. Junge, D. O'Shea, J. Volz, and A. Rauschenbeutel, Phys. Rev. Lett. 110, 213604 (2013).

[13] B. J. M. Hausmann, B. J. Shields, Q. Quan, Y. Chu, N. P. de Leon, R. Evans, M. J. Burek, A. S. Zibrov, M. Markham, D. J. Twitchen, H. Park, M. D. Lukin, and M. Loncăr, Nano Lett. 13, 5791 (2013).

[14] M. Arcari, I. Söllner, A. Javadi, S. Lindskov Hansen, S. Mahmoodian, J. Liu, H. Thyrrestrup, E. H. Lee, J. D. Song, S. Stobbe, and P. Lodahl, Phys. Rev. Lett. 113, 093603 (2014).

[15] I. Söllner, S. Mahmoodian, S. L. Hansen, L. Midolo, G. Kirsanske, T. Pregnolato, H. El-Ella, E. H. Lee, J. D. Song, S. Stobbe, and P. Lodahl, Nat. Nanotechnol. 10, 775 (2015).

[16] R. Mitsch, C. Sayrin, B. Albrecht, P. Schneeweiss, and A. Rauschenbeutel, Nat. Commun. 5, 5713 (2014).

[17] R. J. Coles, D. M. Price, J. E. Dixon, B. Royall, E. Clarke, P. Kok, M. S. Skolnick, A. M. Fox, and M. N. Makhonin, Nat. Commun. 7, 11183 (2016).

[18] S. V. Lobanov, S. G. Tikhodeev, N. A. Gippius, A. A. Maksimov, E. V. Filatov, I. I. Tartakovskii, V. D. Kulakovskii, T. Weiss, C. Schneider, J. Geßler, M. Kamp, and S. Höfling, Phys. Rev. B 92, 205309 (2015).

[19] C. W. J. Beenakker, D. P. DiVincenzo, C. Emary, and M. Kindermann, Phys. Rev. Lett. 93, 020501 (2004).

[20] R. Ionicioiu, Phys. Rev. A 75, 032339 (2007).
[21] S. Fan, S. E. Kocabaş, and J.-T. Shen, Phys. Rev. A 82, 063821 (2010).

[22] See Supplemental Material at http://link.aps.org/ supplemental/10.1103/PhysRevLett.117.240501 contains detailed calculations of parity-measurement and gate fidelities.

[23] A. Gilchrist, N. K. Langford, and M. A. Nielsen, Phys. Rev. A 71, 062310 (2005).

[24] S. Das, A. Grankin, I. Iakoupov, E. Brion, J. Borregaard, R. Boddeda, I. Usmani, A. Ourjoumtsev, P. Grangier, and A. S. Sørensen, Phys. Rev. A 93, 040303 (2016).

[25] N. Somaschi, V. Giesz, L. De Santis, J. C. Loredo, M. P. Almeida, G. Hornecker, S. L. Portalupi, T. Grange, C. Anton, J. Demory, C. Gomez, I. Sagnes, N. D. Lanzillotti Kimura, A. Lemaitre, A. Auffeves, A. G. White, L. Lanco, and P. Senellart, Nat. Photonics 10, 340 (2016).

[26] C. Matthiesen, M. Geller, C. H. H. Schulte, C. L. Gall, J. Hansom, Z. Li, M. Hugues, E. Clarke, and M. Atatüre, Nat. Commun. 4, 1600 (2013).

[27] Y.-M. He, Y. He, Y.-J. Wei, D. Wu, M. Atatüre, C. Schneider, S. Höfling, M. Kamp, C.-Y. Lu, and J.-W. Pan, Nat. Nanotechnol. 8, 213 (2013).

[28] A. V. Kuhlmann, J. Houel, A. Ludwig, L. Greuter, D. Reuter, A. D. Wieck, M. Poggio, and R. J. Warburton, Nat. Phys. 9, 570 (2013).

[29] K. Konthasinghe, J. Walker, M. Peiris, C. K. Shih, Y. Yu, M. F. Li, J. F. He, L. J. Wang, H. Q. Ni, Z. C. Niu, and A. Muller, Phys. Rev. B 85, 235315 (2012).

[30] F. Morichetti, C. Ferrari, A. Canciamilla, and A. Melloni, Laser Photonics Rev. 6, 74 (2012).

[31] C. Simon and W. T. M. Irvine, Phys. Rev. Lett. 91, 110405 (2003).

[32] L.-M. Duan and H. J. Kimble, Phys. Rev. Lett. 90, 253601 (2003).

[33] Y. Akihama, Y. Kanamori, and K. Hane, Opt. Express 19, 23658 (2011).

[34] C. H. Bennett, G. Brassard, S. Popescu, B. Schumacher, J. A. Smolin, and W. K. Wootters, Phys. Rev. Lett. 76, 722 (1996).

[35] D. Deutsch, A. Ekert, R. Jozsa, C. Macchiavello, S. Popescu, and A. Sanpera, Phys. Rev. Lett. 77, 2818 (1996).

[36] H.-J. Briegel, W. Dür, J. I. Cirac, and P. Zoller, Phys. Rev. Lett. 81, 5932 (1998).

[37] L.-M. Duan and R. Raussendorf, Phys. Rev. Lett. 95, 080503 (2005).

[38] S. D. Barrett and P. Kok, Phys. Rev. A 71, 060310 (2005).

[39] T. C. Ralph, A. J. F. Hayes, and A. Gilchrist, Phys. Rev. Lett. 95, 100501 (2005).

[40] D. Brunner, B. D. Gerardot, P. A. Dalgarno, G. Wüst, K. Karrai, N. G. Stoltz, P. M. Petroff, and R. J. Warburton, Science 325, 70 (2009).

[41] A. Delteil, S. Zhe, W.-b. Gao, E. Togan, S. Faelt, and A. Imamoglu, Nat. Phys. 12, 218 (2016).

[42] J. Dreiser, M. Atatüre, C. Galland, T. Müller, A. Badolato, and A. Imamoglu, Phys. Rev. B 77, 075317 (2008).

[43] Q. Wang, S. Stobbe, and P. Lodahl, Phys. Rev. Lett. 107, 167404 (2011).

[44] G. Fernandez, T. Volz, R. Desbuquois, A. Badolato, and A. Imamoglu, Phys. Rev. Lett. 103, 087406 (2009). 
[45] Y. L. Delley, M. Kroner, S. Faelt, W. Wegscheider, and A. İmamoğlu, arXiv:1509.04171.

[46] M. Kroner, K. M. Weiss, B. Biedermann, S. Seidl, S. Manus, A. W. Holleitner, A. Badolato, P. M. Petroff, B. D. Gerardot, R. J. Warburton, and K. Karrai, Phys. Rev. Lett. 100, 156803 (2008).

[47] A. Faraon, C. Santori, Z. Huang, V. M. Acosta, and R. G. Beausoleil, Phys. Rev. Lett. 109, 033604 (2012).
[48] B. Pingault, J. N. Becker, C. H. H. Schulte, C. Arend, C. Hepp, T. Godde, A. I. Tartakovskii, M. Markham, C. Becher, and M. Atatüre, Phys. Rev. Lett. 113, 263601 (2014).

[49] L. J. Rogers, K. D. Jahnke, M. H. Metsch, A. Sipahigil, J. M. Binder, T. Teraji, H. Sumiya, J. Isoya, M. D. Lukin, P. Hemmer, and F. Jelezko, Phys. Rev. Lett. 113, 263602 (2014). 